\title{
Dosimetry of conformal dynamic arc radiotherapy and intensity modulated radiotherapy in unresectable cholangiocarcinoma
}

Thanarpan Peerawonga ${ }^{\mathrm{a}}$, Chonlakiet Khorprasert ${ }^{\mathrm{b}}$, Sivalee Suriyapee ${ }^{\mathrm{b}}$, Taweap Sanghangthum ${ }^{\mathrm{c}}$, Isra Israngkul Na Ayuthaya ${ }^{c}$, Kanjana Shotelersuk ${ }^{\mathrm{b}}$

aDivision of Therapeutic Radiology and Oncology, Department of Radiology, Faculty of Medicine, Prince of Songkla University, Songkhla 90110; ${ }^{b}$ Division of Radiation Oncology, Department of Radiology, Faculty of Medicine, Chulalongkorn University, Bangkok 10330; 'Division of Radiation Oncology, Department of Radiology, King Chulalongkorn Memorial Hospital, Bangkok 10330, Thailand

\begin{abstract}
Background: Radiotherapy in cholangiocrcinoma has to overcome organ tolerance of the upper abdomen. Hi-technology radiotherapy may improve conformity and reduce dose to those organ.

Objective: Quantitatively compare the dosimetry of conformal dynamic arc radiotherapy (CD-arcRT) and intensity modulated radiotherapy (IMRT) in unresectable cholangiocarcinoma.

Material and methods: Eleven cases of unresectable cholangiocarcinoma were re-planned with IMRT and CDarcRT at King Chulalongkhorn Memorial Hospital between 20 September 2004 and 31 December 2005. Both the planning techniques were evaluated using the dose volume histogram of the planning target volume and organ at risk. The conformation number and dose to critical normal structures were used to determine the techniques. Results: IMRT technique was significantly conformed to the planning target volume than CD-arcRT in term of conformation number. For critical structure, IMRT significantly reduced the radiation dose to liver in terms of mean liver dose, V30Gy and V20Gy of the right kidney.

Conclusion: The advantage of IMRT was more conformity and reduced dose to critical structure compared with CD-arcRT, but there was no difference between these techniques in terms of V20Gy of left kidney and maximum dose to the spinal cord.
\end{abstract}

Keywords: Conformal dynamic arc radiotherapy, cholangiocarcinoma, dosimetry; Intensity modulated radiotherapy, radiotherapy

Cholangiocarcinoma is a major problem of cancer treatment in Thailand. Especially, Khon Kaen in the northeastern region of Thailand shows the highest incidence of cholangiocarcinoma in the world [1].

Clinical features of cholangiocarcinoma depend on anatomical location of tumors, intrahepatic,

Correspondence to: Thanarpan Peerawong, MD, Div. of Therapeutic Radiology and Oncology, Department of Radiology, Faculty of Medicine, Prince of Songkla university, Hatyai, Songkhla, 90110, Thailand. E-mail: pthanarpan@gmail.com or extrahepatic lesions. Most patients present with obstructive jaundice. Radical surgery with histologically negative resection margins is the only treatment to cure the patient [2]. When the tumor is surgically unresectable, the prognosis is very poor with median survival only 8-11 months [3].

Chemoradiation is recommended for unresectable cases. The conventional radiation technique, three or four field technique, is usually employed with tumor dose around 45-50 Gy in five-six weeks. The limitation of conventional radiotherapy technique is a low tolerance of organ in upper abdomen [4]. 
Nowadays, there are many advanced and sophisticated radiation treatment techniques, such as three-dimensional radiotherapy, conformal dynamic arc radiotherapy (CD-arcRT), and intensity modulated radiotherapy (IMRT). An advantage of these techniques is increases in radiation dose to tumors and a dose reduction to critical structures.

The conformal radiotherapy technique reported by Aoki et al. [5] is as application of the axial tomography for radiation therapy. This technique was advanced into the dynamic arc conformal therapy. In CD-arcRT, multileaf collimator with gantry angle is dynamically moved around patients to produce multiple conformal radiation fields. Consequently, it reduces dose to normal tissue while integrated higher dose throughout the tumor volume at the same time. This technique achieves steep dose gradient between target volume and the surrounding normal tissue. Treatment time and monitor unit of CD-arcRT are not different from convention rotation radiation techniques, and CD-arcRT has an advantage over IMRT in term of treatment time each day.

In general, IMRT derives a set of intensitymodulated beams by optimizing a pre-defined objective function. An intensity-modulated beam is usually delivered using computer-controlled multileaf collimators, by forming a window that sweeps across the radiation field. The incident beam is divided into multiple of beamlets where intensity varies from 0 $100 \%$ independent of all beams. Dose distributions are determined by the weights of the beamlets and other beam parameters such as beam orientations, couch indices, and collimator angles. Inverse planning process of IMRT is an appropriate technique for irregular shaped tumors, especially the concaved one. IMRT usually takes 20-30 minutes treatment for each conventional fraction.

These modern techniques are capable of increasing radiation dose to tumors located near normal structures with low radiation tolerance. Recently, Cheng et al. [6] reported that IMRT could significantly improve mean tumor dose, reducing dose to spinal cord compared with three-dimensional conformal radiotherapy in hepatoma. Milano et al. [7] compared IMRT with conventional RT in pancreatic and bile duct malignancy, and showed that IMRT reduced the mean dose to liver, stomach, and small bowel.

There are several reports on dosimetry of CDarcRT [5, 8], but their results in cholangiocarcinoma has not been reported yet. In this study, we quantitatively compared dosimetry of CD-arcRT and IMRT in unresectable cholangiocarcinoma. This comparative study would provide us useful information for selecting appropriate radiation technique for our patients.

\section{Materials and methods}

This study was approved by Ethics Committee of the Faculty of Medicine, Chulalongkorn University. The unresectable cholangiocarcinoma patients who underwent CT simulation at King Chulalongkhorn Memorial Hospital, Bangkok between September 20, 2004 and December 12, 2005 were included into the study. The patients were in supine position with the hands raised overhead. The slice of CT image was 5 mm thickness in the entire areas of liver and left and right kidneys. The images were used for tumor and normal structure delineation and re-planned for both CD-arcRT and IMRT.

The flowchart of research methodology is shown in Fig. 1.

\section{Target volume definitions}

Gross tumor volume (GTV) was delineated slice by slice on contrast enhancement CT images, which were registered with the non contrast CT images. If there was non-contrast enhancement study on CT simulation, the gross tumor volume would be delineated by comparison with previous diagnostic CT or magnetic resonance imaging. Clinical target volume (CTV) is defined as GTV plus one cm margin in all direction. Planning target volume (PTV) is defined as CTV plus $0.5 \mathrm{~cm}$ margin in anterior-posterior, right - left lateral and $1.5 \mathrm{~cm}$ in the cranial-caudal axis.

Spinal cord volume was delineated to include whole spinal canal. Liver contour included visualized liver, porta hepatis but excluding gross tumor volume and gall bladder. The left and right kidneys contour was also delineated.

\section{Machine and treatment planning}

Both IMRT and CD-arcRT techniques were applied to each patient by Eclipse Planning System Version 6 (Varian Medical system, Palo Alto, USA).

The treatment machine was Varian Clinac 23 EX, leaf width of $5 \mathrm{~mm}$ for $20 \times 20 \mathrm{~cm}^{2}$ inner field and 10 $\mathrm{mm}$ for the rest of the field. Both techniques utilized $6 \mathrm{MV}$ photon. The dose of $50 \mathrm{~Gy}$ in 25 fractions were prescribed to the planning target volume. To minimize 


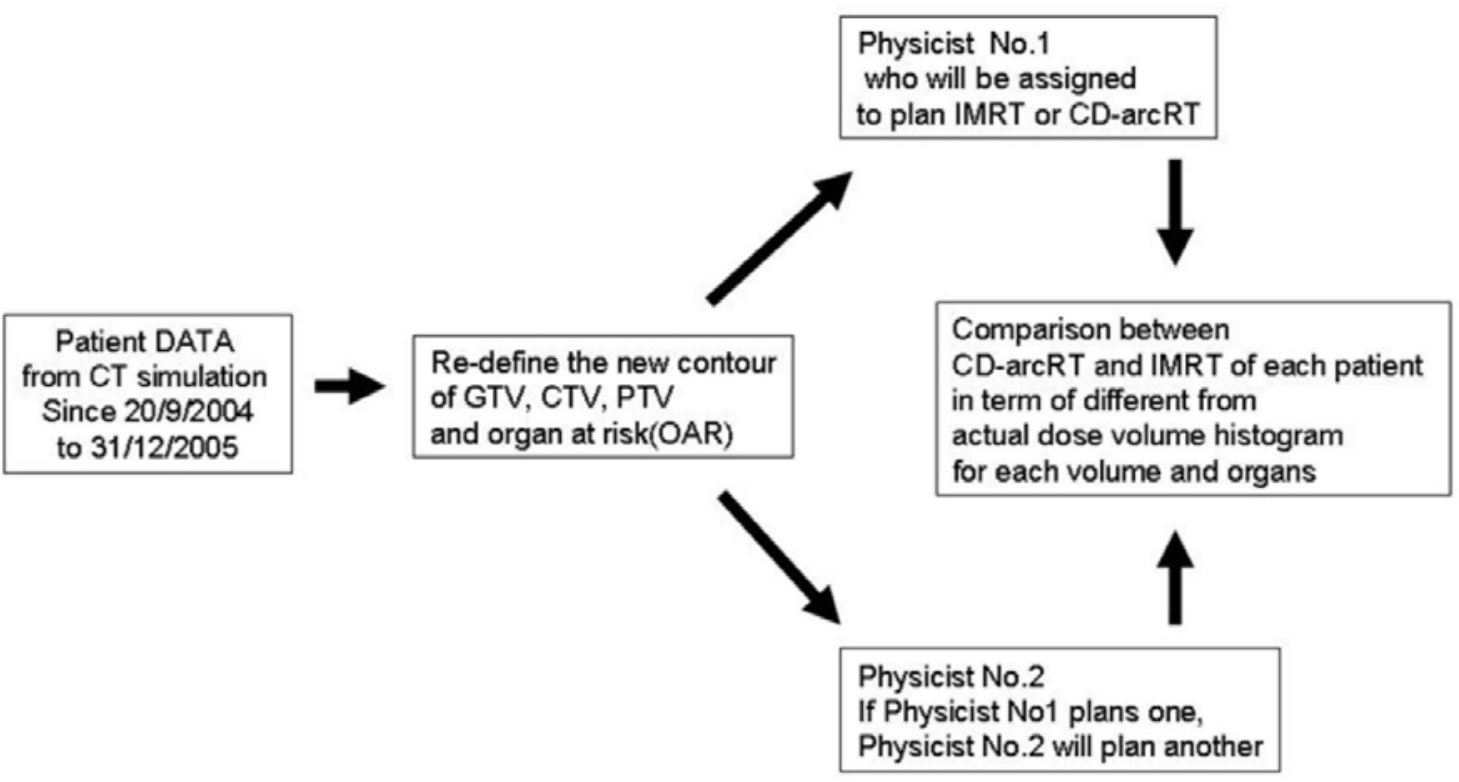

Fig.1 Flowchart of research methodology.

the bias from performing treatment planning, IMRT and CD-arcRT plans were randomized to two physicists.

For CD-arcRT planning, the dynamic multileaf collimator was calculated every two degrees of gantry angle, started at 181 degree clockwise to 180 degree with $6 \mathrm{MV}$ photon beams. For IMRT planning, fivefields $(0,72,144,216$, and 288 degree gantry angle) of six MV photon beams with dMLC were employed. The dose specification and dose volume constraint for IMRT planning were shown in Table1.

After planning for each case, the isodose was normalized to $95 \%$ of the planning target volume and the $100 \%$ isodose line was selected for the prescribed dose. The dose distributions of PTV, dose-volume histograms (DVH) of the PTV and organ at risk (OARs) were evaluated.

\section{Comparison of IMRT to CD-arcRT plan}

To compare the result of IMRT and CD-arc RT plan, we evaluated conformation number (CN) of the target volume and dose volume histogram (DVH) of normal structures including liver, kidneys, and spinal cord.

Conformation number was used to evaluate the conformity of the dose to target volume and normal tissue. Target volume was determined in term of conformity index ( $\left.\mathrm{TV}_{\mathrm{RI}} / \mathrm{TV}\right)$ and healthy tissue was also determined in term of healthy tissue conformity index $\left(\mathrm{TV}_{\mathrm{RI}} / \mathrm{V}_{\mathrm{R}}\right)$, where $\mathrm{TV}_{\mathrm{RI}}$ is target volume cover

Table 1. Dose volume constraint of planning target volume (PTV) and organs at risk (OAR).

\begin{tabular}{llll}
\hline Target volume & Dose & Maximum dose (Gy) & Minimum dose \\
\hline Target volume & 50 Gy in 25 fractions & $110 \%$ of prescribed dose & 95\% of prescribed dose \\
\hline Organ at risk & Volume & Maximum dose (Gy) & \\
\hline Liver & $100 \%$ & 30 \\
Spinal cord & $0 \%$ & 45 & \\
Kidney & $0 \%$ & 20 & \\
If one side of & $10 \%$ & 18 & \\
kidney $>20$ Gy & & & \\
other side & & & \\
\hline
\end{tabular}


by reference dose, $\mathrm{TV}$ is target volume, and $\mathrm{V}_{\mathrm{RI}}$ is volume of reference dose. The conformation number (CN) is derived using the Van't Riet equation [9] as follows:

$$
\mathrm{CN}=\left(\mathrm{TV}_{\mathrm{RI}} / \mathrm{TV}\right) \times\left(\mathrm{TV}_{\mathrm{RI}} / \mathrm{V}_{\mathrm{R}}\right)
$$

Concerning organ at risk, liver doses were determined in terms of mean dose and the percentage of the liver volume that received dose at $30 \mathrm{~Gy}$ (V30Gy). The percentage of the kidneys volume which received the radiation dose at 20Gy in each kidney was also studied. Maximum radiation dose of spinal cord, at least one $\mathrm{cm}^{3}$ of spinal cord volume received that dose, was also determined.

\section{Statistical analysis}

Paired t-test was employed to determine the difference of two techniques.

\section{Results}

Out of 40 cases of cholangiocarcinoma patients referred for radiation treatment at Division of Therapeutic Radiology and Oncology, King Chulalongkhorn Memorial Hospital, only 14 cases were unresectable cholangiocarcinoma. Three cases were excluded from this study because the tumor and normal tissue could not be clearly delineated due to CT artifact. Eleven cases were included into the analysis. Patient characteristics, tumor, and normal liver volume are shown in Table 2.
Most patients were male with hilar type cholangiocarcinoma. Two cases in this study were simulated using 4-D CT simulation. We subsequently used maximum intensity projection (MIP) images for delineation and calculation. The example of isodose distribution and DVHs comparison were shown in Fig. 2 and 3, respectively.

\section{Dose-volume analysis}

Conformation of planning target volume. The average planning target volume doses were $49.99 \mathrm{~Gy}$ for both IMRT and CD-arcRT. The average of maximum dose and minimum dose for IMRT and CDarcRT were shown in Table 3. The average maximum and average minimum dose for CD-arcRT were higher than IMRT with p-value of 0.004 and 0.002 , respectively. Regarding conformation number, the average CN of IMRT and CD-arcRT were 0.93 (range: 0.95 to 0.90 , SD: 0.02 ) and 0.76 (range: 0.83 to 0.68 , SD: 0.05 ), respectively. Diagram of CN of IMRT and CD-arc RT of eleven patients was illustrated in Fig. 4.

Dose to organ at risk. The mean dose to normal liver was 24.2 Gy for IMRT and 26.7 Gy for CD-arc RT, p 0.001 . The mean normal liver doses were more than 30Gy in four cases of CD-arcRT. Those were 31.66Gy, 32.81Gy, 31.02Gy, and 31.03Gy, which were not found in IMRT. The mean volumes of liver receiving radiation in IMRT were significantly lower than those in CD-arcRT in terms of V30Gy (see Table 3).

Table 2. Patient characteristics.

\begin{tabular}{|c|c|c|c|c|c|c|}
\hline No & SEX & Age & Location & $\begin{array}{l}\text { PTV } \\
\left(\mathrm{cm}^{3}\right)\end{array}$ & $\begin{array}{l}\text { Normal liver } \\
\text { Volume }\left(\mathrm{cm}^{3}\right)\end{array}$ & Remark \\
\hline 1 & M & 48 & Hilar & 351.2 & 1855.4 & \\
\hline 2 & M & 40 & Hilar & 382.2 & 2432.3 & MIP \\
\hline 3 & M & 46 & Hilar & 272.6 & 987.9 & \\
\hline 4 & M & 41 & Hilar & 360.2 & 2546.2 & \\
\hline 5 & M & 50 & right hepatic lobe & 666.2 & 1774.1 & MIP \\
\hline 6 & M & 54 & Hilar & 451.6 & 1651 & \\
\hline 7 & $\mathrm{~F}$ & 52 & Hilar & 394.3 & 1481.4 & \\
\hline 8 & $\mathrm{~F}$ & 50 & Hilar & 508.1 & 1519.6 & \\
\hline 9 & $\mathrm{~F}$ & 51 & Distal extrahepatic & 181.8 & 1259.1 & \\
\hline 10 & M & 57 & Distal extrahepatic & 375.5 & 1051 & \\
\hline 11 & $\mathrm{M}$ & 68 & Hilar & 224.6 & 1715.9 & \\
\hline \multicolumn{4}{|c|}{ Average } & 379 & 1,651 & \\
\hline
\end{tabular}

M: male, F: female, PTV: planning target volume, MIP: maximum intensity projection. 

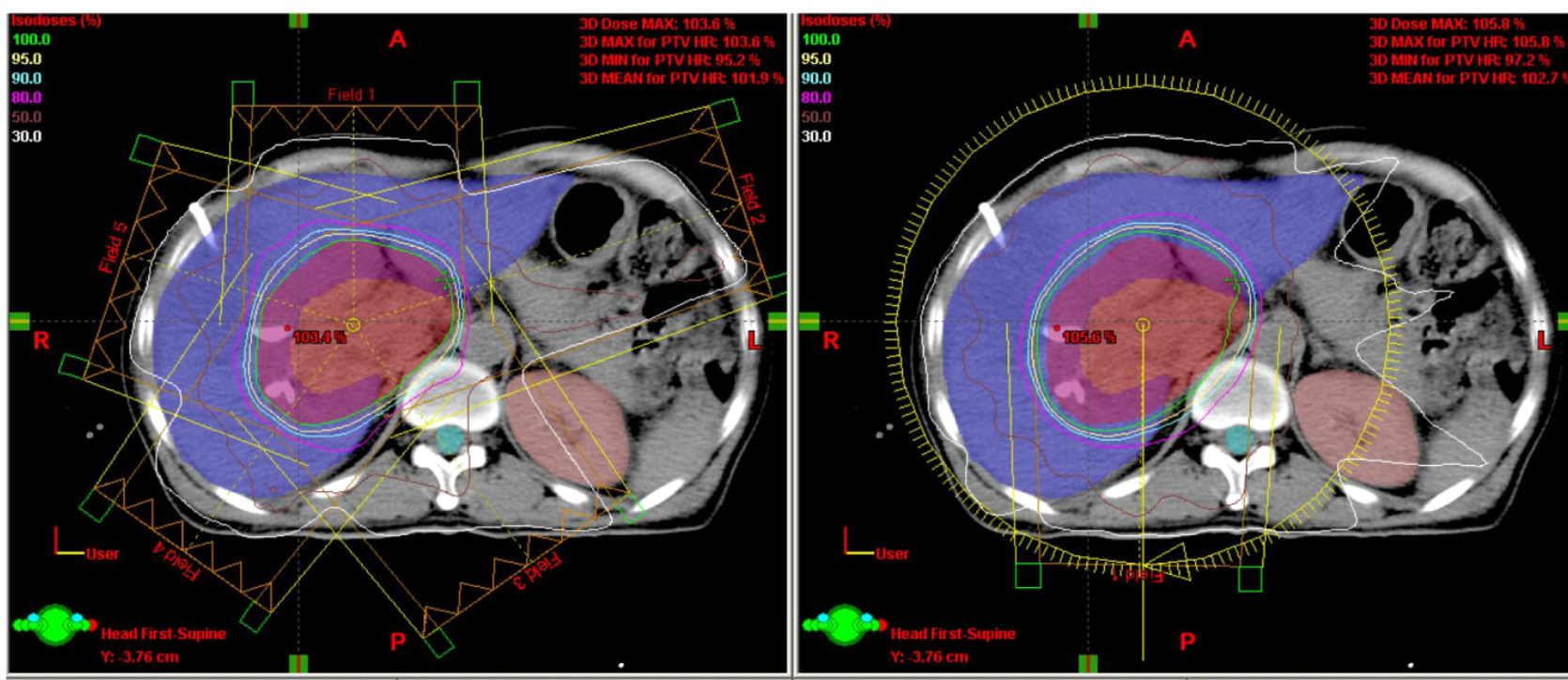

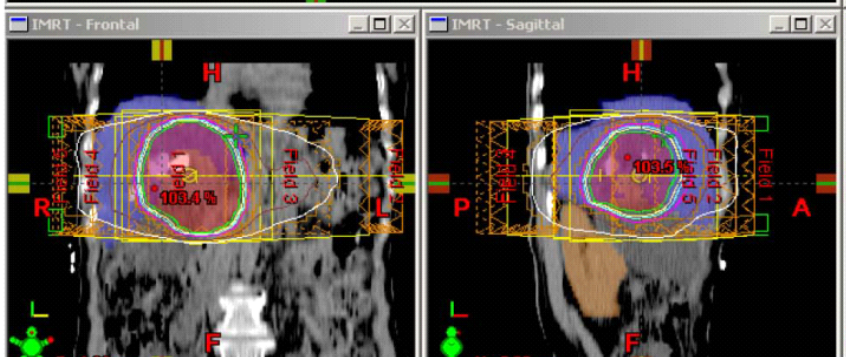

Intensity modulated radiotherapy

(IMRT)

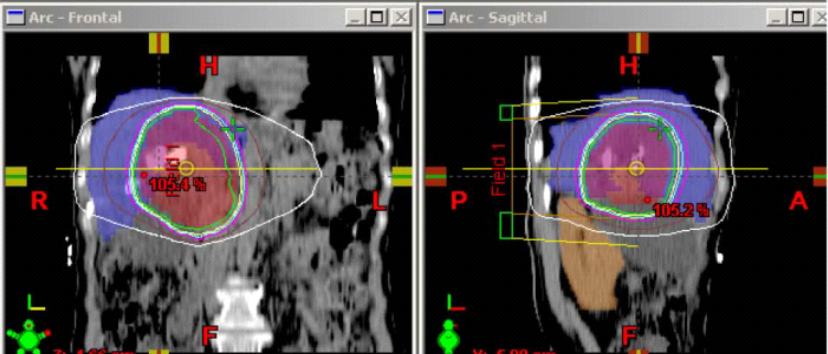

Conformal dynamic arc radiotherapy (CD-arcRT)

Fig. 2 Comparison of isodose distribution of patient No.3.

Both whole kidneys received radiation dose less than 20Gy. The V20Gy of right kidney were $20.28 \%$ in IMRT and $36.71 \%$ in CD-arcRT ( $\mathrm{p}=0.015$ ). Whereas the V20Gy of left kidney were $0.31 \%$ and $4.36 \%(\mathrm{p}=0.082)$ in IMRT and CD-arc RT, respectively.

The maximum doses of spinal cord were 27.9Gy and 25.9Gy for IMRT and CD-arcRT respectively. There was no significant difference between the two techniques.

\section{Discussion}

We reported comparison of two techniques of radiation therapy (IMRT and CD-arcRT) in unresectable cholangiocarcinoma. By normalizing $95 \%$ of the planning target volume in the treatment planning, the $100 \%$ isodose line was selected for the prescribed dose. The target volume conformity index of IMRT and CD-arcRT had no statistically significant difference. However, the significant difference in conformation number was contributed from the difference of healthy tissue conformity index of these two techniques. The healthy tissue conformity indices of CD-arcRT were lower than those of IMRT. These were explained from the physical properties of treatment planning. Although the CD-arcRT achieves steep dose gradient between target volume and the surrounding normal tissue, the isodose distribution is usually spherical-shaped this is not suitable for irregular parts of PTV, as shown in Fig. 2. Therefore, in irregular lesions, it is likely that IMRT produces better conformation to both target volume and healthy normal tissue. In addition, CD-arcRT is fast-to-plan, fast-totreat and utilize lower monitor unit comparing to IMRT. At present, a new and advanced technology, volumetric modulated arc therapy, is being introduced to diminish some weaknesses of IMRT. This radiation technique combines the benefit of IMRT and CDarcRT. The treatment time is fast and conformation as IMRT techniques. The results of volumetric modulated arc therapy have been reported in prostate and lung cancer $[10,11]$. The volumetric modulated 


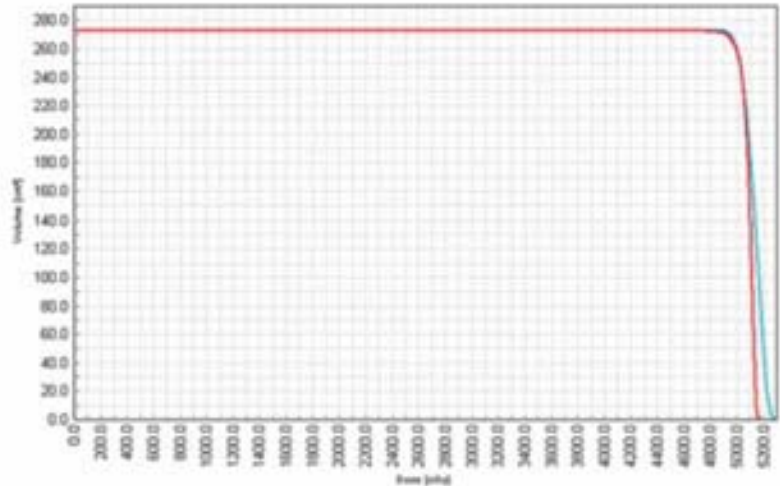

DVHs of PTV

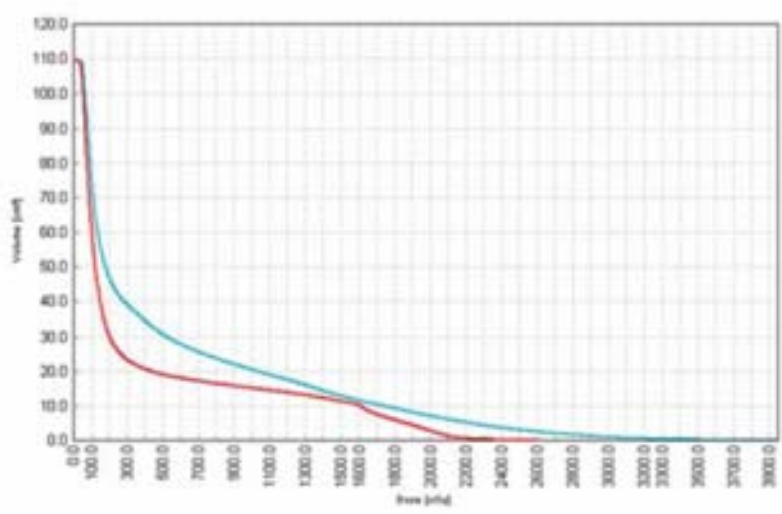

DVHs of right kidney

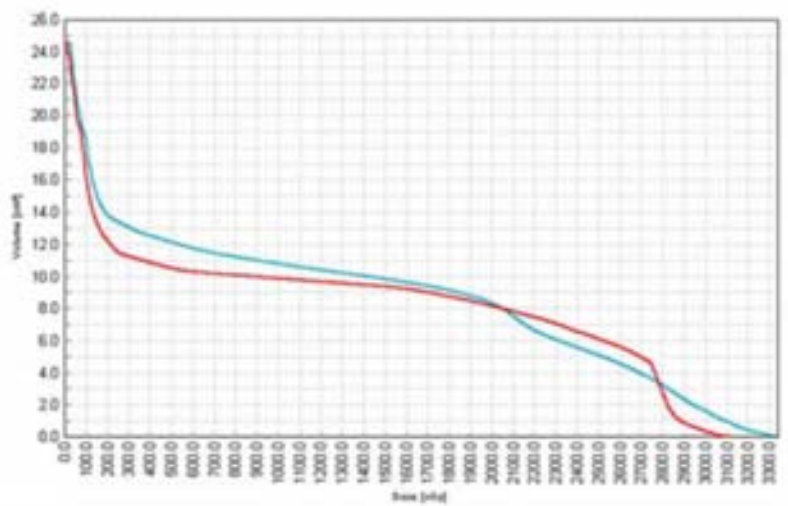

DVHs of spinal cord

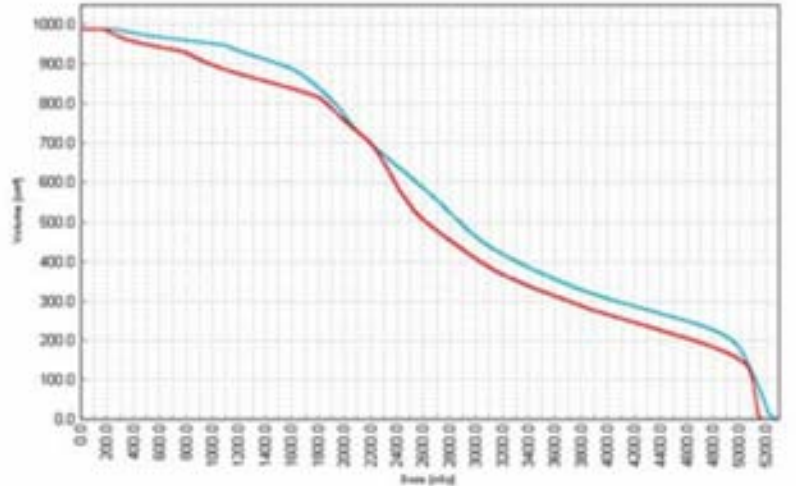

DVHs of normal liver

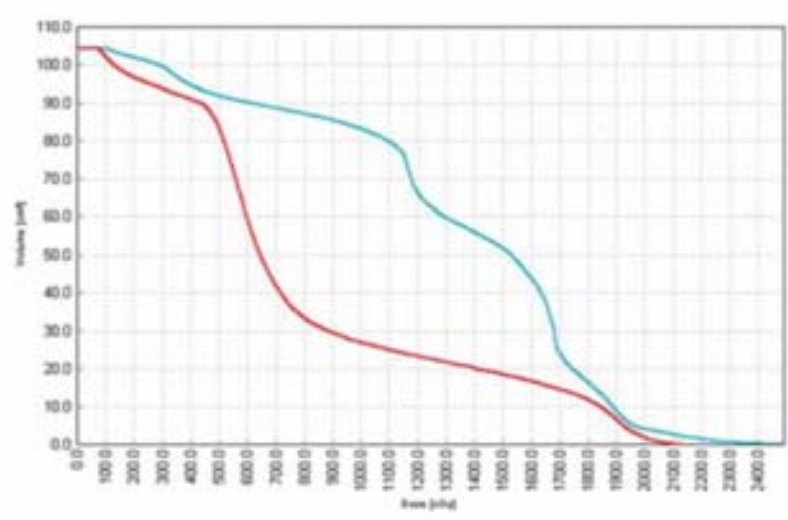

DVHs of left kidney

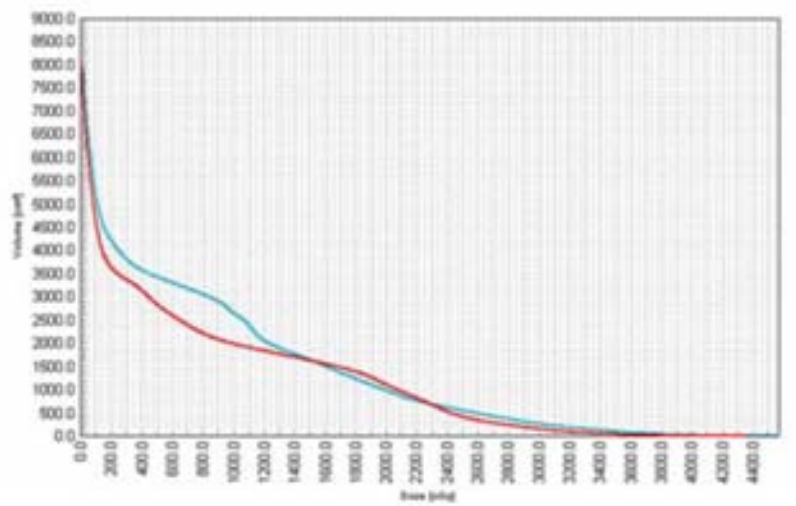

DVHs of tissue outside PTV

Fig. 3 Dose volume histograms (DVHs) of patient No.3 in comparison of IMRT and CD-arcRT, where cyan and red line represent IMRT and CD-arcRT, respectively. 
Table 3. Radiation dose to planning target volume (PTV), conformation number (CN) and dose to critical structures in both techniques.

\begin{tabular}{|c|c|c|c|c|c|}
\hline \multirow[t]{2}{*}{ Parameters } & \multicolumn{2}{|c|}{ IMRT } & \multicolumn{2}{|c|}{ CD-arcRT } & \multirow{2}{*}{$\begin{array}{l}\text { P-value } \\
\text { of pair t-test }\end{array}$} \\
\hline & Range & Average & Range & Average & \\
\hline \multicolumn{6}{|l|}{ Planning target volume } \\
\hline Maximum dose (Gy) & 51.58-54.01 & 52.63 & 52.64-56.07 & 53.90 & 0.004 \\
\hline Minimum dose (Gy) & 48.27-49.21 & 48.78 & 48.50-49.53 & 49.28 & 0.002 \\
\hline Conformation number & $0.90-0.95$ & 0.93 & $0.68-0.83$ & 0.76 & 0.000 \\
\hline Conformity index & $0.95-0.95$ & 0.95 & $0.95-0.95$ & 0.95 & 0.043 \\
\hline Healthy tissue conformity index & $0.94-1.00$ & 0.98 & $0.72-0.87$ & 0.81 & 0.000 \\
\hline \multicolumn{6}{|l|}{ Critical structure } \\
\hline \multicolumn{6}{|l|}{ Liver } \\
\hline Mean dose (Gy) & 18.05-29.8 & 24.2 & $21.0-32.8$ & 26.7 & 0.001 \\
\hline V30Gy & $16.6-41.2$ & 28.6 & 23.4-53.4 & 35.8 & 0.001 \\
\hline \multicolumn{6}{|l|}{ Right Kidney } \\
\hline V20Gy & $0-84.8$ & 20.3 & $0.3-83.3$ & 36.7 & 0.015 \\
\hline \multicolumn{6}{|l|}{ Left Kidney } \\
\hline V20Gy & $0-1.6$ & 0.3 & $0-21.1$ & 4.3 & 0.082 \\
\hline \multicolumn{6}{|l|}{ Spinal cord } \\
\hline Maximum & $19.7-35.9$ & 27.9 & $16.7-35.3$ & 25.9 & 0.139 \\
\hline Radiation dose at vol. 1 cc (Gy) & & & & & \\
\hline
\end{tabular}

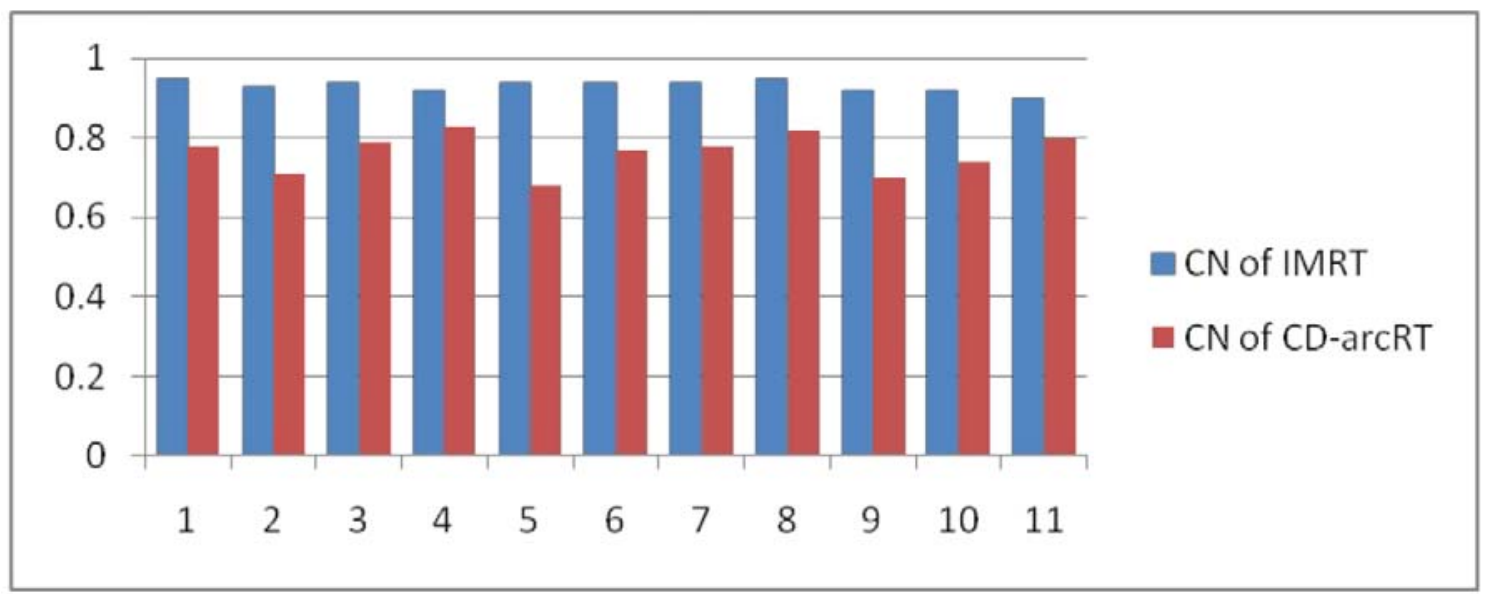

Fig. 4 Comparison of conformation number of IMRT and CD-arcRT.

arc therapy appears interesting in unresectable cholangiocarcinoma.

In this study, parameters to determine liver doses were used from Kim et al. [12] who studied radiationinduced liver disease [12]. Comparing to CD-arcRT, IMRT significantly reduced dose to livers in terms of mean liver dose, V25Gy, V30Gy, V35Gy, and V40Gy, the dose and volume of organs at risk, compared with other studies of upper abdomen (Table 4). Our study demonstrated comparable result with other studies.
Comparing to IMRT and 3-D CRT in hepatocellular carcinoma by Cheng et al [6], the mean liver doses were 28.15Gy for IMRT and 24.06Gy for 3D-CRT [6]. However, the mean liver doses in our study were higher than those from the study by Milano et al. [7] regarding dose distribution in pancreatic and bile duct cancer. This is because of the location of PTV of pancreatic cancer is outside normal liver and more posterior. Considering V30Gy of liver, the results showed no difference to other reports. 
Table 4. Summary of actual dose of organs at risk (OAR) of malignancy in upper abdomen in other studies.

\begin{tabular}{llllllll}
\hline Authors & $\begin{array}{l}\text { Target } \\
\text { volume }\end{array}$ & Techniques & $\begin{array}{l}\text { Mean } \\
\text { liver } \\
\text { dose } \\
\text { (Gy) }\end{array}$ & $\begin{array}{l}\text { Liver } \\
\text { V30Gy }\end{array}$ & $\begin{array}{l}\text { V20Gy } \\
\text { of RK }\end{array}$ & $\begin{array}{l}\text { V20Gy } \\
\text { of LK }\end{array}$ & $\begin{array}{l}\text { Maximum } \\
\text { dose to } \\
\text { spinal cord }\end{array}$ \\
\hline Cheng, et al. [6] & Hepatoma & IMRT & 28.15 & $44 \%$ & NR & NR & NR \\
Milano, et al. [7] & Pancreas & 3D-CRT & 24.06 & $42 \%$ & NR & NR & NR \\
& and bile duct & IMRT & 19.3 & $25 \%$ & $25 \%$ & $13 \%$ & NR \\
& & 3D4F & 23.4 & $36 \%$ & (V22Gy) & (V22Gy) & NR \\
Milano, et al. [13] & Gastric cancer & IMRT & 44.6 & $19 \%$ & $12 \%$ & $52 \%$ & NR \\
& & 3D2F & 41.6 & $34 \%$ & $20 \%$ & $67 \%$ & NR \\
Present study & Bile duct & 3D3F & 67.9 & $64 \%$ & $21 \%$ & $52 \%$ & NR \\
& & IMRT & 24.2 & $29 \%$ & $20 \%$ & $0.31 \%$ & 27.9 \\
& & CD-arcRT & 26.8 & $36 \%$ & $37 \%$ & $4.3 \%$ & 25.9
\end{tabular}

NR: not report, RK: right kidney, LK: left kidney, IMRT: intensity modulated radiotherapy, 3D-CRT: three dimension conformal radiotherapy, 3D4F: three-dimension conformal radiotherapy with four field technique, 3D2F: three-dimension conformal radiotherapy with two fields technique, 3D3F: three-dimension conformal radiotherapy with three field technique.

Most bilateral kidneys were spared in most studies treating upper abdomen, but in the study regarding dose distribution of gastric cancer, some parts of the left kidney was included in the target volume [13]. For this reason, the dose to the left kidney might be higher than that in other studies. By evaluating dose to the kidney using V20Gy, we found that the right kidney dose was higher than left kidney. The kidney doses from CD-arcRT were significantly higher than doses from IMRT, 37\% vs. 20\% in the right kidney $(\mathrm{p}=0.015)$. The V20Gy of the left kidney was slightly higher in CD-arcRT, 4.3\% compared with $0.3 \%$ in IMRT.

Since the spinal cord is a serial organ, we evaluated its dose using by the maximum dose to cover $1 \mathrm{~cm}^{3}$ volume of the spinal cord. The average maximum dose of spinal cord was less than organ tolerance in both techniques.

\section{Conclusion}

IMRT and CD-arcRT techniques provided satisfactory isodose distribution in unresectable cholangiocarcinoma. IMRT provided better conformation number and reduced dose to the liver and right kidneys compared with CD-arcRT. Both techniques can preserve the left kidney. The maximum doses of the spinal cord were not different.

The authors have no conflicts of interest to report.

\section{References}

1. Vatanasapt V, Sriamporn S, Vatanasapt P. Cancer control in Thailand. Jpn J Clin Oncol. 2002; 32:S82-91.

2. Urego M, Flickinger JC, Carr BI. Radiotherapy and multimodality management of cholangiocarcinoma. Int J Radiat Oncol Biol Phys. 1999; 44:121-6.

3. Crane CH, Macdonald KO, Vauthey JN, Yehuda P, Brown T, Curley S, et al. Limitations of conventional doses of chemoradiation for unresectable biliary cancer. Int J Radiat Oncol Biol Phys. 2002; 53:969-74.

4. Emami B, Lyman J, Brown A, Coia L, Goitein M, Munzenrider JE, et al. Tolerance of normal tissue to therapeutic irradiation. Int J Radiat Oncol Biol Phys. 1991;21:109-22.

5. Aoki T, Nagata Y, Mizowaki T, Kokubo M, Negoro Y, Takayama K, et al. Clinical evaluation of dynamic arc conformal radiotherapy for paraaortic lymph node metastasis. Radiother Oncol. 2003; 67:113-8.

6. Cheng JC, Wu JK, Huang CM, Liu HS, Huang DY, Tsai SY, et al. Dosimetric analysis and comparison of threedimensional conformal radiotherapy and intensitymodulated radiation therapy for patients with hepatocellular carcinoma and radiation-induced liver disease. Int J Radiat Oncol Biol Phys. 2003; 56:229-34.

7. Milano MT, Chmura SJ, Garofalo MC, Rash C, Roeske JC, Connell PP, et al. Intensity-modulated radiotherapy in treatment of pancreatic and bile duct malignancies: toxicity and clinical outcome. Int J Radiat Oncol Biol Phys. 2004; 59:445-53. 
8. Verellen D, Linthout N, Soete G, Van Acker S, De Roover P, Storme G. Considerations on treatment efficiency of different conformal radiation therapy techniques for prostate cancer. Radiother Oncol. 2002; 63:27-36.

9. Feuvret L, Noel G, Mazeron JJ, Bey P. Conformity index: a review. Int J Radiat Oncol Biol Phys. 2006; 64:333-42.

10. Bedford JL, Nordmark Hansen V, McNair HA, Aitken AH, Brock JE, Warrington AP, et al. Treatment of lung cancer using volumetric modulated arc therapy and image guidance: A case study. Acta Oncol. 2008; 25: $1-6$.

11. Palma D, Vollans E, James K, Nakano S, Moiseenko V, Shaffer R, et al. Volumetric modulated arc therapy for delivery of prostate radiotherapy: comparison with intensity-modulated radiotherapy and threedimensional conformal radiotherapy. Int J Radiat Oncol Biol Phys. 2008; 72:996-1001.

12. Kim TH, Kim DY, Park JW, Kim SH, Choi JI, Kim HB, et al. Dose-volumetric parameters predicting radiationinduced hepatic toxicity in unresectable hepatocellular carcinoma patients treated with three-dimensional conformal radiotherapy. Int J Radiat Oncol Biol Phys. 2007; 67:225-31.

13. Milano MT, Garofalo MC, Chmura SJ, Farrey K, Rash C, Heimann R, et Intensity-modulated radiation therapy in the treatment of gastric cancer: early clinical outcome and dosimetric comparison with conventional techniques. Br J Radiol. 2006; 79:497-503. 